\title{
Pre-operative evaluation of circulating KL-6 levels as a biomarker for epithelial ovarian carcinoma and its correlation with tumor MUC1 expression
}

\author{
SHO SATO ${ }^{1,2}$, TOMOMI KATO ${ }^{3}$, KENJI ABE ${ }^{4}$, TATSUYA HANAOKA ${ }^{1,2}$, YURI YANO $^{1}$, AKIRA KUROSAKI ${ }^{1,2}$, \\ MASANORI YASUDA $^{3}$, TETSUO SEKINO ${ }^{4}$, KEIICHI FUJIWARA ${ }^{1,2}$ and KOSEI HASEGAWA ${ }^{1,2}$ \\ ${ }^{1}$ Department of Gynecologic Oncology, Saitama Medical University International Medical Center; \\ ${ }^{2}$ Gynecologic Oncology Translational Research Unit, Project Research Division, Research Center for Genomic Medicine, \\ Saitama Medical University; ${ }^{3}$ Department of Pathology, Saitama Medical University International Medical Center, Hidaka, \\ Saitama 350-1298; ${ }^{4}$ Department of Research and Development, Eidia Co., Ltd, Inashiki, Ibaraki 300-1155, Japan
}

Received July 23, 2016; Accepted February 17, 2017

DOI: $10.3892 / 01.2017 .6254$

\begin{abstract}
Krebs von den Lungen-6 (KL-6), a mucinous sialylated sugar chain on human mucin-1 glycoprotein (MUC1), is a diagnostic marker for interstitial lung diseases. Furthermore, elevated serum KL-6 levels have been observed in certain malignant tumor types of epithelial origin. The expression of MUC1 has been observed in patients with epithelial ovarian cancer (EOC) and is considered a potential therapeutic target. In the present study, KL- 6 serum levels were investigated in patients clinically suspected of having malignant ovarian tumors. A total of 219 patients were enrolled in the study, which analyzed their serum KL-6 levels in addition to tumor expression of MUC1 using immunohistochemistry. High serum KL-6 levels were predominantly observed in patients with EOC, and did not occur in patients with benign or borderline tumors. The level of serum KL-6 was highly correlated with tumor stage, grade and histological type, and demonstrated superior sensitivity for the detection of ovarian cancer compared with that of serum cancer antigen 125. High serum KL-6 was significantly associated with shorter progression-free survival. In addition, tumor MUC1 expression status was significantly correlated with serum KL-6 levels. These data suggest that serum KL-6 may be a useful, non-invasive
\end{abstract}

Correspondence to: Dr Kosei Hasegawa, Department of Gynecologic Oncology, Saitama Medical University International Medical Center, 1397-1 Yamane, Hidaka, Saitama 350-1298, Japan E-mail: koseih@saitama-med.ac.jp

Abbreviations: KL-6, Krebs von den Lungen-6; MUC1, human mucin-1 glycoprotein; ILD, interstitial lung disease; FFPE, formalin-fixed paraffin-embedded; mAb, monoclonal antibody; ROC, receiver operator characteristic; EOC, epithelial ovarian cancer; PFS, progression-free survival; OS, overall survival

Key words: ovarian cancer, Krebs von den Lungen-6, human mucin-1 glycoprotein, serum biomarker, diagnostic marker biomarker surrogate for tumor MUC1 expression in future clinical trials of MUC1-targeted therapy.

\section{Introduction}

Ovarian cancer is the fifth leading cause of cancer-associated mortality for women in the USA (1). It is the most fatal type of gynecologic malignancy, and is characterized by few early symptoms, widespread peritoneal dissemination and ascites at advanced stages (2). Existing limitations in the detection, diagnosis and treatment of ovarian cancer have contributed to a poor overall survival (OS) rate (2). It is therefore imperative that such difficulties are overcome. The majority of epithelial ovarian cancer (EOC) cases $(61 \%)$ are diagnosed in the advanced stages, and the mean 5-year survival rate is $27 \%$ (2). However, $60-90 \%$ of patients diagnosed when they are at stage I/II of the disease survive, depending on the tumor grade; thus, there is an increased possibility for curing the disease in cases of earlier detection and treatment $(3,4)$. Since physical symptoms are almost absent in the early stages of ovarian cancer, efforts to develop assays for blood or urine based non-invasive biomarkers are currently in progress (5). Serum cancer antigen (CA)125 is an ovarian cell surface glycoprotein that has been demonstrated to be elevated in $80 \%$ of patients with advanced EOC (6). However, in the early stages of EOC, CA125 possesses a predictive value of $10 \%$ (7). This can be attributed to the relatively low specificity of CA125. Elevated CA125 levels have been associated with various non-malignant conditions, including pregnancy, endometriosis, adenomyosis, uterine fibroids, pelvic inflammatory disease, menstruation and benign ovarian cysts (8). Furthermore, abnormal CA125 levels have been associated with other malignancies, including pancreatic (9), breast (10), lung (11), gastric (12) and colorectal cancer (13), particularly when they are associated with peritoneal spreading. CA125 may not be useful in identifying early stages or providing a differential diagnosis for these diseases, as malignancies with peritoneal spreading typically exhibit a high level of CA125. However, CA125 may be suitable for monitoring the response 
of patients to chemotherapy and in the early detection of relapse in patients with ovarian cancer (14).

Human mucin-1 glycoprotein (MUC1) is encoded by the MUC1 gene, which is located on the long arm (q) of chromosome 1 at position 21, and has a molecular weight between 250 and $500 \mathrm{kDa}$. The transmembrane glycoprotein MUC1 is a well-known target for cancer therapy (15). MUC1 is aberrantly glycosylated and overexpressed in $>90 \%$ of epithelial cancer types, including ovary, breast, pancreas and lung epithelial cancer (16). Several studies have investigated the association between the expression of MUC1 and EOC (17), and have demonstrated that MUC1 is overexpressed in $>90 \%$ of late-stage EOC and metastatic lesions (18). MUC1 expression is typically observed in the glandular or luminal epithelial cells of the mammary gland, esophagus, stomach, duodenum, pancreas, uterus, prostate and lungs, and in hematopoietic cells to a lesser extent (15). MUC1 possesses negatively charged sugar branches that are extended such that a physical barrier is created, conferring an anti-adhesive property to MUC1 that limits accessibility and prevents microbial growth $(19,20)$. Such biological differences between tumor and healthy tissue makes MUC1 a promising therapeutic target for cancer, particularly in the context of targeting MUC1 for tumor-specific killing.

MUC1 is currently being investigated in preclinical and clinical trials as a diagnostic and therapeutic target for ovarian cancer $(21,22)$. Full-length MUC1 is comprised of two subunits, the N-terminal (MUC1-N) and C-terminal (MUC1-C); in normal growth conditions, MUC1 remains as a heterodimer (MUC1-N and MUC-C) on the cell surface (15). Soluble MUC1 is generated from cleavage of the extracellular domain by enzymes such as disintegrin and metalloprotease domain-containing protein 17 and matrix metalloproteases $(23,24)$. Cleaved MUC1-N has been identified in the circulation of patients with cancer, and can be used as a biomarker for cancer diagnosis, staging or monitoring relapse following initial therapy (15,23-25).

Krebs von den Lungen-6 (KL-6) is a high-molecular-weight glycoprotein classified as 'cluster 9' (MUC-associated) according to the Third International Workshop on Lung Tumor and Differentiation Antigens (26). The anti-KL-6 monoclonal antibody (mAb) is considered to recognize the specific MUC1 glycopeptide sequence, but the precise glycan structure of the epitope remains unclear (27). In a study by Seko et al (28), it was reported that newly-identified O-linked glycans of MUC1, containing 6'-sulfo-galactose/ $\mathrm{N}$-acetylgalactosamine, could be the carbohydrate epitopes of the anti-KL-6 mAb. KL- 6 was first suggested as a serum tumor marker for pulmonary, breast and pancreatic cancer; however, the diagnostic accuracy of KL-6 was demonstrated to be lower than carcinoembryonic antigen levels, based on the high number of false positives in patients with pulmonary fibrosis (29). Further investigations have demonstrated that KL-6 is elevated in patients with interstitial pneumonia (30). KL-6 has been approved by Japan's National Health Insurance Program as a diagnostic marker for interstitial lung diseases (ILDs) since 1999; presently, KL-6 levels are examined in $>2,000,000$ samples annually in Japan (29). The results of a study performed by Nakao et al (31) identified an association between increased KL-6 levels and ovarian cancer with predominantly intrathoracic lesions. This suggests that KL-6 is a useful tumor marker for ovarian cancer. Thus, further studies are warranted on the diagnostic and prognostic value of KL-6 as a tumor biomarker for EOC in a prospective clinical setting with detailed patient information.

In the present study, there was focus on patients who were clinically suspected to have malignant ovarian tumors, and the serum KL-6 levels were investigated in these patients prior to surgery. Serum KL-6 was evaluated as a diagnostic marker for ovarian cancer and was compared with CA125. Furthermore, tumor MUC1 expression was immunohistochemically examined to investigate the association between circulating KL-6 and tumor MUC1 status. Thus, the results of the present study address the feasibility of using this non-invasive test in future clinical studies of MUC1-targeted therapies for patients with EOC.

\section{Materials and methods}

Patients, sera and tumor specimens. The present study was a retrospective analysis, based on a previous prospective study for ovarian cancer biomarkers that was reviewed and approved by the Institutional Review Board of Saitama Medical University International Medical Center (Saitama, Japan); full details are described in Kurosaki et al (32). In brief, 219 women were enrolled in the study prior to surgery at Saitama Medical University International Medical Center between December 2010 and March 2013. The patients were clinically suspected of having borderline-to-malignant ovarian tumor, and were thus investigated to validate candidate soluble molecules for ovarian cancer biomarkers. All patients eventually underwent surgery. Of the 219 patients, 132 (56.9\%), 40 $(17.3 \%)$ and $43(18.6 \%)$ patients were pathologically diagnosed with malignant, borderline and benign ovarian tumors, respectively. Furthermore, 4 patients were diagnosed with ovarian metastasis of a primary colorectal cancer. Of the 132 malignant ovarian tumors, 126 tumors were diagnosed with EOC. Of these, 60 (47.5\%) were serous carcinoma, 22 (17.5\%) endometrioid carcinoma, $34(27.0 \%)$ clear cell carcinoma, 5 (4.0\%) mucinous carcinoma and 5 were classed as 'others'. There were 38 cases of EOC (30.2\%) at stage I, 21 (16.6\%) at stage II, $54(42.9 \%)$ at stage III and $13(10.3 \%)$ at stage IV.

Tissue specimens were collected at the time of surgery, and formalin-fixed paraffin-embedded (FFPE) specimens were stored at room temperature. Patient sera were collected from all patients prior to surgery and stored immediately at $-80^{\circ} \mathrm{C}$ until required for use in subsequent experiments. Serum CA125 levels were measured at the Central Clinical Laboratory of Saitama Medical University. Relevant clinical and histopathological information were extracted from clinical charts and pathology reports, respectively. The clinical stage of each tumor was determined according to the guidelines provided by the International Federation of Gynecology and Obstetrics (FIGO) staging system (33). The histological type and grade of each tumor were determined by pathologists experienced in gynecologic oncology (Department of Pathology, Saitama Medical University International Medical Center, Saitama, Japan).

Measurement of serum KL-6. Sera from 219 patients were available for the present study. Serum levels of KL-6 were examined using a Picolumi KL-6 kit (EIDIA Co., Ltd., Ibaraki, 
Japan), which is a Japan Pharmaceuticals and Medical Devices Agency-approved electrochemiluminescence immunoassay kit (approval no. 21100AMZ00542000). All assay steps were performed in accordance with the manufacturer's protocol and were performed using anonymously coded samples by technicians who were blind to the patients' diagnosis.

Immunohistochemical staining. The NCL-MUC1 antibody (clone Ma695; Leica Microsystems, Ltd., Milton Keynes, UK), a specific antibody that binds to the carbohydrate epitope of the human MUC1 glycoprotein, was used to investigate tumor MUC1 expression in FFPE specimens obtained from 158 EOC tissues, including borderline malignancies. Immunohistochemical staining was performed on slides with $5 \mu \mathrm{m}$ sections using a BenchMark XT Automated Slide Stainer and an iVIEW DAB Detection kit, according to the manufacturer's protocol (both from Ventana Medical Systems, Inc., Tucson, AZ, USA). Sections were first deparaffinized and hydrated. Subsequently, antigen retrieval was performed using Cell Conditioning Solution 1 (Ventana Medical Systems, Inc.), followed by incubation for $1 \mathrm{~h}$ with the aforementioned mouse monoclonal anti-MUC1 primary antibody (1:100 dilution) at room temperature, and incubation for $0.5 \mathrm{~h}$ with the aforementioned iVIEW DAB Detection kit at for $0.5 \mathrm{~h}$ at $37^{\circ} \mathrm{C}$. Staining was observed under a light microscope and was considered positive if membranous or membranous with cytoplasmic stain was present in tumor cells. Slides were then scored according to the proportion of staining as follows: Negative, $<1 \% ; 1+,<25 \% ; 2+,<75 \%$; and $3+, \geq 75 \%$. Furthermore, a score of $2+$ or $3+$ was classified as high tumor MUC1 expression. The scoring of MUC1 staining was blindly evaluated by two independent observers. Any discrepancies were resolved by a joint review using a double-headed microscope.

Statistical analysis. One-way analysis of variance (ANOVA) with Tukey's post hoc analysis, Student's $t$-tests and Mann-Whitney U tests were used to assess the differences between the patient groups. Receiver operator characteristic (ROC) curve analysis was performed to determine the differences between sensitivity and specificity for serum KL-6 or CA125 between the patient groups. The cut-off values for serum KL- 6 or CA125 were determined using the Youden's index as the maximum value of (sensitivity+specificity-1). This index was calculated from all points of an ROC curve and used as a criterion for selecting the optimum cut-off value. Survival was assessed using the Kaplan-Meier estimator method, and any significant differences between groups were determined using the log-rank test. All statistical analyses were performed using GraphPad Prism software (version 6.0; GraphPad Software, Inc., La Jolla, CA, USA). P $<0.05$ (two-tailed) was considered to indicate a statistically significant difference.

\section{Results}

Serum KL-6 in patients with malignant, borderline and benign ovarian tumors. A total of 219 patients were enrolled in the present study prior to surgery. These patients were clinically suspected of having borderline-to-malignant ovarian tumors, and eventually underwent surgical intervention (Fig. 1A). Sera from all patients were analyzed for serum KL-6 levels. As illustrated in Fig. 1B, patients with malignant tumors exhibited high levels of serum KL-6 while patients with benign or borderline tumors exhibited low levels of serum KL-6. Despite the small sample size $(n=4)$, patients with ovarian metastasis from colorectal cancer did not exhibit elevated serum KL-6 levels. The median serum KL-6 levels in those with malignant ovarian tumors was $450.5 \mathrm{U} / \mathrm{ml}$ [95\% confidence interval (CI), 330-890 U/ml], which was significantly higher compared with that of borderline $(202.5 \mathrm{U} / \mathrm{ml} ; 95 \%$ CI, 189.0-224.0 U/ml) and benign tumors (205.0 U/ml; 95\% CI, 180.0-252.0 U/ml) $(\mathrm{P}<0.01$, Tukey's test). Low levels of serum KL-6 were observed in almost all non-epithelial ovarian tumor serum samples (data not shown).

Association between serum KL-6 and clinicopathological factors in patients with epithelial ovarian, fallopian tube and primary peritoneal cancer. Table I summarizes the serum KL-6 levels in association with the clinicopathological features in 126 patients with epithelial cancer. Of these patients, $108 \mathrm{had}$ primary ovarian cancer, 12 had primary fallopian tube cancer and 6 had primary peritoneal cancer. High serum KL-6 levels were identified to be correlated with FIGO stage, histological type, tumor grade, lymph node metastasis and residual tumor size. Patients with cancer in the advanced stages exhibited significantly higher serum KL-6 levels compared with those exhibited by patients in the early stage $(\mathrm{P}=0.0018)$. Serum KL-6 levels were significantly higher in serous tumor serum samples compared with those of the non-serous histotype $(\mathrm{P}=0.0028$, Student's $t$-test). Grade 3 tumors exhibited significantly higher serum KL-6 levels compared with those of grade 1 and 2 tumors $(\mathrm{P}<0.01$, Tukey's test). Furthermore, high serum KL-6 levels were observed in patients with lymph node metastases $(\mathrm{P}=0.0011)$. A residual tumor size of $\geq 1 \mathrm{~cm}$ demonstrated higher serum KL-6 levels compared with those with no residual tumor size $(\mathrm{P}<0.001$, Tukey's test). No significant correlation was identified between serum KL-6 levels and other clinical variables.

Serum KL-6 as a diagnostic marker for EOC. The diagnostic significance of serum KL-6 in differentiating patients with EOC from patients with pelvic masses suspected as malignant ovarian tumors was investigated. The ability of serum KL-6 in predicting the presence of EOC was compared with that of CA125. Fig. 2A demonstrates the ROC curves for all patients to predict EOC, and Table II summarizes those results. The ROC analysis indicated that serum KL-6 was marginally superior to CA125, with areas under the curve (AUCs) of 0.81 and 0.78 for serum KL-6 and CA125, respectively. Youden's index, a measure that serves as a summary of the ROC curve (34), also indicated that serum KL-6 performed better than CA125, with Youden's index values of 0.55 and 0.48 for serum KL- 6 and CA125, respectively. The appropriate cut-off values determined by the Youden's index for serum KL- 6 and CA125 to predict EOC were 293 and $225 \mathrm{U} / \mathrm{ml}$, respectively. Regarding EOC, the diagnostic sensitivity, specificity and positive predictive value for serum KL-6 were 70.1, 85.1 and 87.2\%, respectively. However, those for CA125 were 58.6, 89.7 and $88.2 \%$, respectively (Table II). Serum KL-6 thus exhibited an improved performance in discriminating between EOCs and other tumors, with higher sensitivity compared with CA125.

Detecting early stage EOC using a serum marker is often difficult due to its lower sensitivity at early stages of 
Table I. Correlation between KL-6 serum levels and clinicopathological features in epithelial ovarian cancer patients.

\begin{tabular}{|c|c|c|c|}
\hline Clinicopathological factor & $\mathrm{N}(\%)$ & Mean KL-6 (SD), U/ml & P-value \\
\hline \multicolumn{4}{|l|}{ Age, years } \\
\hline$\leq 60$ & $67(53.1)$ & $1,310(1,684)$ & \multirow[t]{2}{*}{$0.3456^{\mathrm{a}}$} \\
\hline$>60$ & $59(46.9)$ & $1,712(2,979)$ & \\
\hline \multicolumn{4}{|l|}{ Menopause } \\
\hline Yes & $89(70.7)$ & $1,571(2,598)$ & \multirow[t]{2}{*}{$0.5931^{\mathrm{a}}$} \\
\hline No & $37(29.3)$ & $1,321(1,761)$ & \\
\hline \multicolumn{4}{|l|}{ FIGO stage } \\
\hline I, II & $59(46.8)$ & $802(2,518)$ & \multirow[t]{2}{*}{$0.0018^{\mathrm{a}}$} \\
\hline III, IV & $67(53.2)$ & $2,111(2,080)$ & \\
\hline \multicolumn{4}{|l|}{ Primary site } \\
\hline Ovary & $108(85.7)$ & $1,438(2,444)$ & \multirow[t]{3}{*}{$0.6723^{\mathrm{b}}$} \\
\hline Fallopian tube & $12(9.5)$ & $1,632(1,313)$ & \\
\hline Peritoneum & $6(4.8)$ & $2,309(2,983)$ & \\
\hline \multicolumn{4}{|l|}{ Histology } \\
\hline Serous carcinoma & $60(47.5)$ & $2,155(1,963)$ & \multirow[t]{5}{*}{$0.0028^{c}$} \\
\hline Endometrioid carcinoma & $22(17.5)$ & $1,254(3,997)$ & \\
\hline Clear cell carcinoma & $34(27.0)$ & $792(1,640)$ & \\
\hline Mucinous carcinoma & $5(4.0)$ & $265(74)$ & \\
\hline Others & $5(4.0)$ & $722(442)$ & \\
\hline \multicolumn{4}{|l|}{ Grade } \\
\hline 1 & $20(15.9)$ & $562(786)$ & \multirow[t]{3}{*}{$0.0013^{\mathrm{b}}$} \\
\hline 2 & $70(55.5)$ & $1,176(1,653)$ & \\
\hline 3 & $36(28.6)$ & $2,645(3,523)$ & \\
\hline \multicolumn{4}{|l|}{ Tumor size, mm } \\
\hline$<100$ & $61(48.4)$ & $1,685(1,936)$ & \multirow[t]{2}{*}{$0.0034^{\mathrm{a}}$} \\
\hline$\geq 100$ & $65(51.6)$ & $1,322(2,733)$ & \\
\hline \multicolumn{4}{|l|}{ Ascites cytology } \\
\hline Positive & $78(61.9)$ & $1,730(1,907)$ & \multirow[t]{2}{*}{$0.1627^{\mathrm{a}}$} \\
\hline Negative & $48(38.1)$ & $1,120(2,974)$ & \\
\hline \multicolumn{4}{|l|}{ Lymph node metastasis } \\
\hline Positive & $17(13.5)$ & $3,227(4,473)$ & \multirow[t]{2}{*}{$0.0011^{\mathrm{a}}$} \\
\hline Negative & $109(86.5)$ & $1,228(1,743)$ & \\
\hline \multicolumn{4}{|l|}{ Residual tumor size, $\mathrm{cm}$} \\
\hline 0 & $60(47.6)$ & $767(1,504)$ & \multirow[t]{3}{*}{$0.0011^{\mathrm{b}}$} \\
\hline$<1$ & $7(5.6)$ & $891(958)$ & \\
\hline$\geq 1$ & $59(46.8)$ & $2,314(2,919)$ & \\
\hline
\end{tabular}

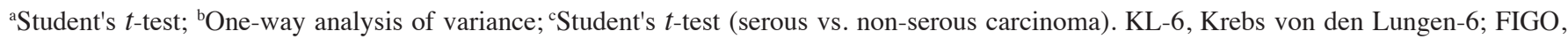
International Federation of Gynecology and Obstetrics; SD, standard deviation.

diseases (35). CA125, as a marker, exhibits a high false-positive rate for benign and borderline tumors (8). Therefore, the levels of serum KL-6 were compared among localized epithelial ovarian tumors, including epithelial benign tumors, borderline tumors and stage I/II EOCs. Based on the ROC analysis in Fig. 2B and Table II, the sensitivity of serum KL-6 and CA125 in diagnosing stage I/II EOC from benign and borderline tumors was compared. It was observed that KL-6 possessed an improved sensitivity compared with that of CA125 in detecting patients with stage I/II EOC. Using KL-6, 50.8\% (30/59) of patients were diagnosed with early stage EOC, while only $32.2 \%$ (19/59) patients were identified to be positive for early stage EOC when CA125 was used. In addition, 35\% (14/40) of patients with stage I/II EOC who were CA125-negative were identified as positive for serum KL-6. Thus, KL-6 may demonstrate complementary expression with CA125 in patients with early stage EOC.

Association between serum KL-6 and survival in patients with EOC. The prognostic potential of serum KL-6 in patients 
Table II. Summary of receiver operator characteristic curve analysis of KL-6 and CA125.

\begin{tabular}{|c|c|c|c|c|}
\hline \multirow[b]{2}{*}{ Factor } & \multicolumn{2}{|c|}{ EOC (all patients) vs. others ${ }^{a}$} & \multicolumn{2}{|c|}{ EOC (stage I /II) vs. others ${ }^{\mathrm{a}}$} \\
\hline & KL-6 & CA125 & KL-6 & CA125 \\
\hline AUC & 0.81 & 0.78 & 0.68 & 0.62 \\
\hline Youden's index & 0.55 & 0.48 & 0.35 & 0.22 \\
\hline Cut-off value, $\mathrm{U} / \mathrm{ml}$ & 293 & 225 & 293 & 224 \\
\hline Sensitivity, \% & 70.1 & 58.6 & 50.8 & 32.2 \\
\hline Specificity, \% & 85.1 & 89.7 & 83.9 & 89.7 \\
\hline PPV, \% & 87.2 & 88.2 & 68.2 & 67.9 \\
\hline NPV, $\%$ & 66.2 & 60.0 & 71.6 & 66.1 \\
\hline Accuracy, $\%$ & 76.1 & 71.2 & 70.5 & 66.4 \\
\hline
\end{tabular}

KL-6, Krebs von den Lungen-6; CA, cancer antigen; EOC, epithelial ovarian cancer; AUC, area under the curve; PPV, positive predictive value; NPV, negative predictive value. ancludes benign and borderline tumors, and ovarian metastases.

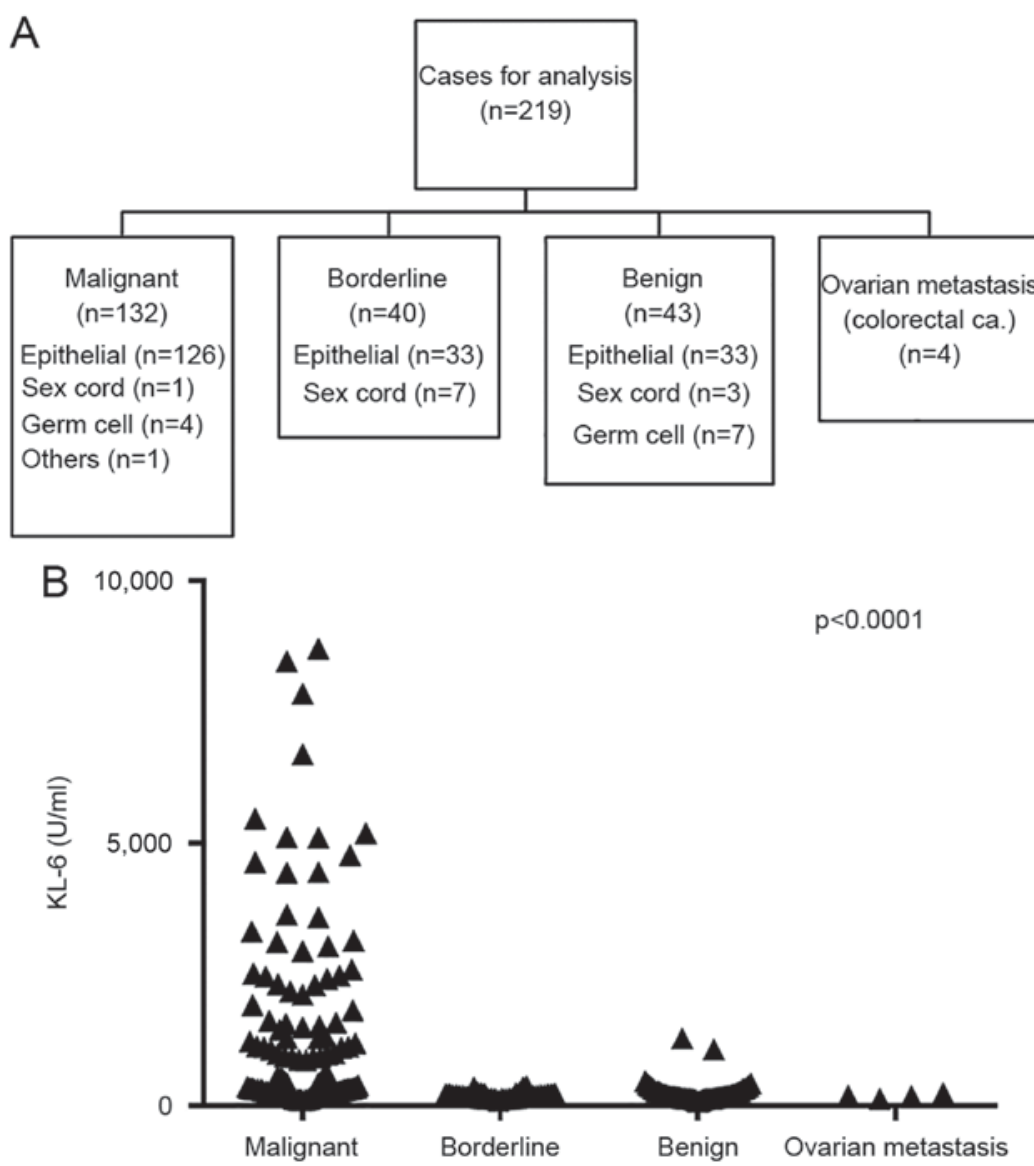

Figure 1. Characteristics of the 219 patients initially included in the study. (A) Diagnoses of the 219 patients who presented with clinical suspicion of a malignant ovarian tumor, as initially included in the present study. (B) Serum KL-6 levels in the types of ovarian tumor, as determined using an electrochemiluminescent immunoassay. One-way analysis of variance was used in the statistical analyses of differences in distribution of KL-6 levels between each group. Ca., cancer; KL-6, Krebs von den Lungen-6.

with EOC was assessed by measuring its levels immediately prior to primary surgery. Of the 126 patients included in the analysis, the median follow-up time for patients who were still alive following the initial diagnosis was 41 months. Compared with patients with higher serum KL-6 levels, patients with lower serum KL-6 levels experienced a significantly longer progression-free survival (PFS) $(\mathrm{P}<0.0001$; Fig. 3A), where serum KL-6 $<514.5 \mathrm{U} / \mathrm{ml}$ was considered low (median, 514.5 U/ml). However, despite optimizing the cut-off points for OS, including average, median and quartiles, no prognostic association between serum KL-6 levels and OS in the population was determined. Survival analyses were performed on 

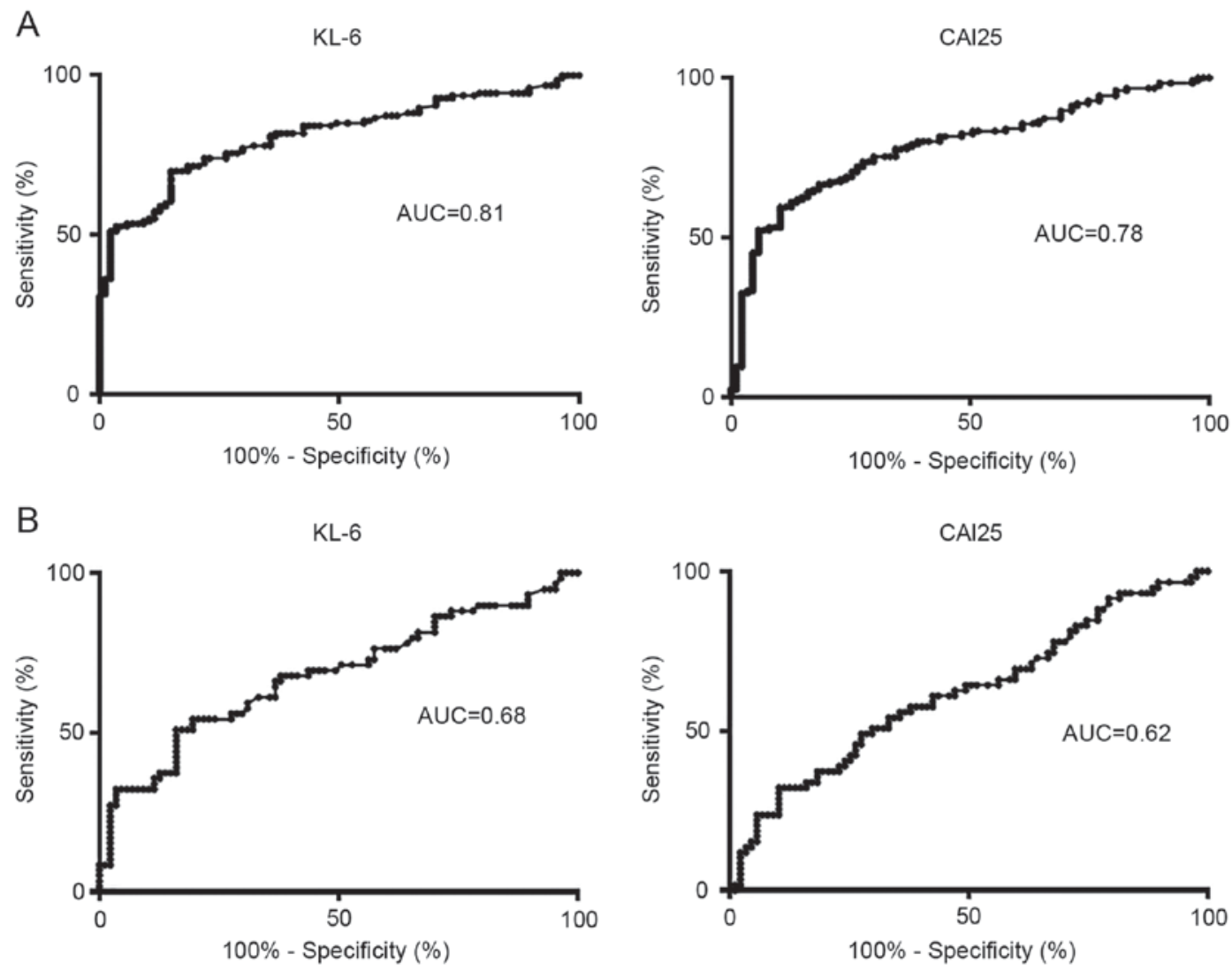

Figure 2. (A) Serum KL-6 and CA125 ROC curves for the prediction of epithelial ovarian, fallopian tube and primary peritoneal cancer. (B) Serum KL-6 and CA125 ROC curves for the prediction of early stage (stages I and II) epithelial ovarian cancer. KL-6, Krebs von den Lungen-6; CA125, cancer antigen 125; AUC, area under the curve; ROC, receiver operator characteristic.

subgroups by stage of disease, as high KL-6 was frequently observed in advanced stage tumors (Table I). As demonstrated in Fig. 3B and C, no correlations between serum KL-6 and clinical outcomes in early and advanced patients with EOC subgroups were identified.

Association between serum KL-6 and tumor MUC1 expression. Several clinical trials are currently investigating the efficacy of MUC1-targeted therapies $(21,22)$. It is important to note, however, that although tumor MUC1 expression is potentially important in patient selection for such targeted therapies, it is often difficult to measure due to limited sample availability, particularly at the time of recurrence (32). In addition, a real-time non-invasive method to monitor the targeted therapy ensures safety and efficacy (36). Consequently, it appeared appropriate to investigate the association between serum KL-6 and tumor MUC1 expression to assess whether serum KL-6 levels could reflect tumor MUC1 status. For this purpose, MUC1 expression in EOC was assessed using immunohistochemistry with a specific antibody that binds to the carbohydrate epitope of the human MUC1 glycoprotein. The representative images demonstrated in Fig. 4A illustrated that tumor MUC1 expression was evident in the majority of EOCs, albeit with variable proportions of staining. Fig. 4B illustrates the association between tumor MUC1 expression and serum KL-6 in the patients with EOC. A correlation was identified, indicating an association between increasing serum KL-6 levels and tumor MUC1 expression status $(\mathrm{P}<0.0001$, one-way ANOVA with Tukey's post hoc test). Thus, scores of 2+ and 3+ were defined as high tumor MUC1 expression, and ROC analysis was performed to determine if serum KL-6 could predict high tumor MUC1 expression (Fig. 5).

Based on the cut-off value of $584 \mathrm{U} / \mathrm{ml}$ calculated (Table III), 0\% (0/9) and 13\% (3/23) of patients with negative and 1+ tumor MUC1 expression, respectively, demonstrated serum KL-6 levels above the cut-off value. However, $46.6 \%$ (27/58) of patients with $2+$ and $80.0 \%$ (28/35) of patients with $3+$ MUC1 expression exhibited positive serum KL-6 results. A cut-off value of $584 \mathrm{U} / \mathrm{ml}$ was able to predict high MUC1 expression with a predictive value of $94.8 \%$ in patients with EOC (Table III). Although 26.5\% (9/34) and 8.8\% (3/34) of patients with borderline malignancy demonstrated $2+$ and $3+$ tumor MUC1 expression following immunohistochemical analysis, respectively, no serum KL-6 was detected (Fig. 1B). These data suggest that circulating KL-6 may predict the local tumor MUC1 status in a non-invasive manner. Notably, serum KL-6 may potentially serve as a surrogate marker for tumor MUC1 expression in patients with EOC.

\section{Discussion}

In the present study, the serum KL-6 levels in patients who were suspected with having malignant ovarian tumors were analyzed. High levels of serum KL-6 were observed in patients with malignant ovarian tumors that did not possess ILDs. However, those with borderline or benign tumors demonstrated 

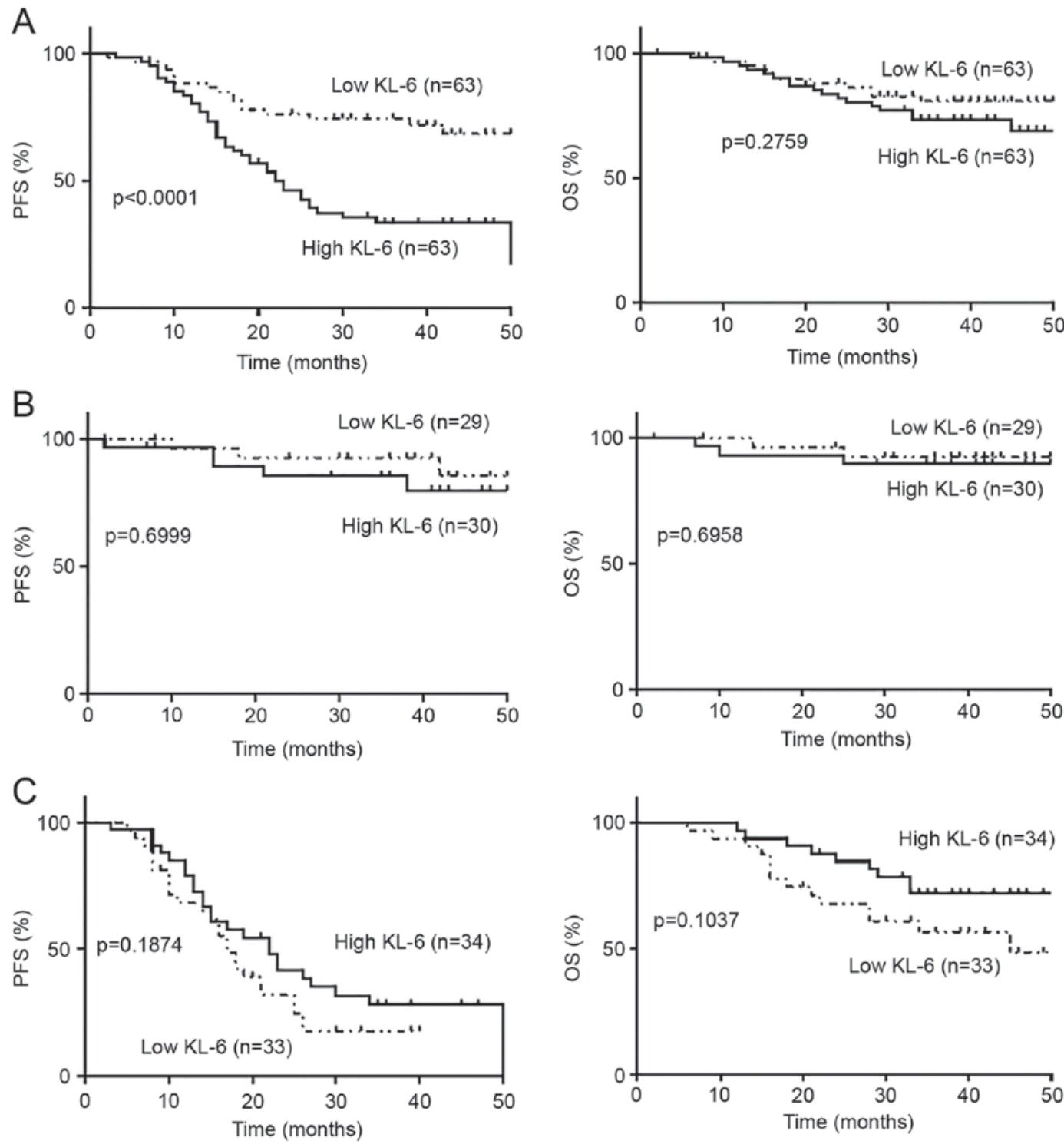

Figure 3. Kaplan-Meier survival curves and corresponding serum KL-6 levels in patients with (A) ovarian cancer in (B) stages I and II and (C) stages III and IV. The log-rank test was used for the statistical analysis of the difference in survival rate between the low and high serum KL-6 groups. The median value ( $515 \mathrm{U} / \mathrm{ml}$ for all patients, $294 \mathrm{U} / \mathrm{ml}$ for the early stage subgroup and 1,459 U/ml for the advanced stage subgroup) was the cut-off value between groups for serum KL-6. PFS was significantly different between the low and high serum KL-6 groups in all patients. No survival difference between the high and low serum KL-6 groups was observed in other subgroups. KL-6, Krebs von den Lungen-6; PFS, progression-free survival; OS, overall survival.

either no increase or a marginal increase in serum KL-6 levels. Elevated serum KL-6 levels correlated with FIGO stage, histological type, tumor grade, tumor size, lymph node metastasis and residual tumor size. The results from the ROC analysis indicated that serum KL-6 was more sensitive in predicting EOC than CA125. In addition, it was demonstrated through immunohistochemical staining that serum KL-6 levels correlated with tumor MUC1 expression. This promising result suggests that circulating KL- 6 could potentially serve as a biomarker for monitoring disease progression or responses to therapeutics in ovarian cancer and even in patient selection for MUC1-targeted therapies.

Ovarian cancer biomarkers remain an important unmet clinical necessity with regard to early disease detection, predicting prognosis and monitoring therapeutic responses (5). CA125 is currently the most commonly used serum biomarker for monitoring ovarian cancer (37). However, its poor specificity has led to high false-positive rates in screening and early disease detection (35). MUC1 is a glycoprotein that is overexpressed in malignant tumor cells, and has been demonstrated to serve an important role in tumor development, growth, invasion, cellular signal transduction and chemoresistance (38). Serum MUC1 has been investigated as a biomarker for breast cancer and EOC based on antibodies directed against circulating MUC1 antigens (17). There are several assays to detect soluble MUC1 using mAbs such as DF3, HMFG1, HMFG2 and M2C5 (17). Serum MUC1 levels can also be measured with the soluble MUC1 antigens, CA15.3 and CA27.29 (39-41). Fisken et al (42) evaluated soluble MUC1 levels using HMFG2 antibody in patients with EOC, and revealed that 45 and $61 \%$ of patients with stage I and III EOC, respectively, had elevated serum MUC1. Furthermore, these levels were identified to be significantly correlated with post-operative residual tumor volume (42). A retrospective study by Havrilesky et al (37) 

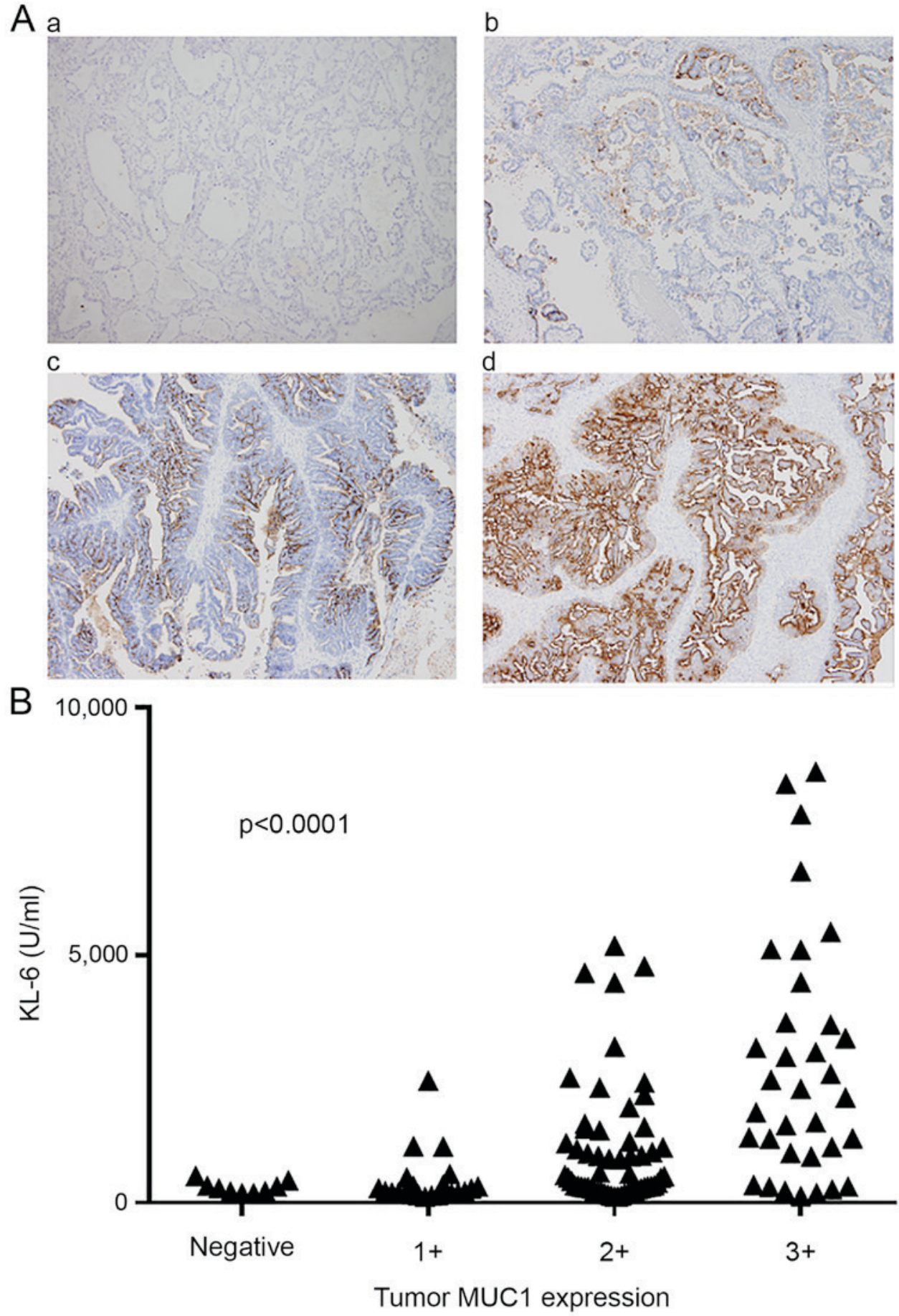

Figure 4. (A) Immunohistochemical staining of tumor MUC1 expression in ovarian cancers (magnification, 10x). Representative specimens are included for (a) negative staining, $<1 \%$ positive staining; (b) $1+$ staining, $<25 \%$ positive staining; (c) $2+$ staining, $<75 \%$ positive staining; and (d) $3+$ staining, $\geq 75 \%$ positive staining. (B) Association between serum KL-6 and tumor MUC1 expression. Serum KL-6 was determined using an electrochemiluminescent immunoassay, and tumor MUC1 expression was scored using immunohistochemical staining. Elevated serum KL-6 levels were frequently observed in tumors with 2+ and 3+ MUC1 expression. One-way analysis of variance and Tukey's post hoc analysis were used for the statistical analyses of differences in KL-6 level distribution between each group. KL-6, Krebs von den Lungen-6; MUC1, human mucin-1 glycoprotein.

reported that when serum MUC1 levels were measured using M2C5, a specific anti-MUC1 mAb, in $200 \mathrm{EOC}$ patients and 396 healthy controls, the sensitivity and specificity for EOC were $\sim 50$ and $75 \%$, respectively, at the optimum cut-off value.

KL-6 is a mucinous sialylated sugar chain on MUC1 (26). Serum KL-6 is elevated in the majority of patients with ILDs, and is widely accepted in Japan as a diagnostic test for ILDs and as a marker of disease activity $(29,30)$. A number of studies have addressed circulating KL-6 in malignant tumors, including lung (43), breast (44) and hepatocellular carcinoma (45). However, to the best of our knowledge, no studies regarding serum KL-6 as a biomarker for EOC have been performed to date. In the present study, serum KL-6 in patients with EOC was retrospectively analyzed, and the results revealed that high serum KL-6 levels were correlated with disease progression. The ROC analysis 
Table III. Summary of receiver operator characteristic curve analysis for predicting high tumor human mucin-1 glycoprotein expression using serum Krebs von den Lungen-6 levels.

\begin{tabular}{lc}
\hline Factor & Value \\
\hline AUC & 0.78 \\
Youden's index & 0.50 \\
Cut-off value, U/ml & 584 \\
Sensitivity, \% & 59.1 \\
Specificity, \% & 90.6 \\
PPV, \% & 94.8 \\
NPV, \% & 43.3 \\
Accuracy, \% & 67.2 \\
\hline
\end{tabular}

AUC, area under the curve, PPV, positive predictive value; NPV, negative predictive value.

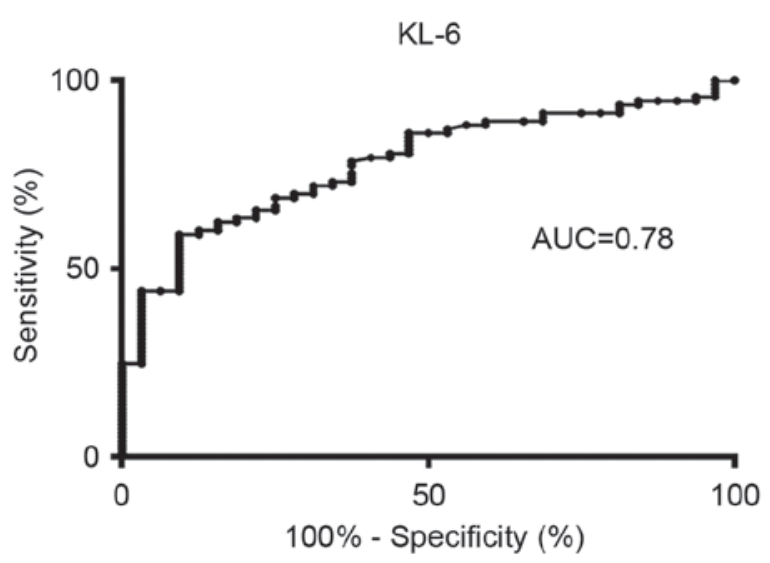

Figure 5. Serum KL-6 receiver operator characteristic curve to predict high tumor human mucin-1 glycoprotein expression as defined by an immunohistochemical staining score of $2+$ and 3+. KL-6, Krebs von den Lungen-6; AUC, area under the curve.

data demonstrated that serum KL-6 levels, as a diagnostic marker, possess a higher sensitivity for EOC than CA125. Furthermore, the data suggest that serum KL-6 is able to distinguish stage I/II EOC from borderline and benign tumors. These results reinforce soluble MUC1 as a useful indicator of EOC, and demonstrate that serum KL-6 serves as a more effective diagnostic marker than CA125. A possible explanation may be that different anti-MUC1 mAbs bind to different epitopes of the MUC1 antigen.

The present study demonstrates serum KL- 6 as prognostic marker for PFS in ovarian cancer, but not for OS. This may be associated with the treatment regimen employed following first relapse as different treatments may have been used between individuals, or reflect the response to frontline chemotherapy. It is also possible that the follow-up period was insufficient to make conclusions regarding OS. Budiu et al (46) reported serum MUC1 (CA15.3) level as a potential prognostic biomarker for platinum-resistant EOC, suggesting that high levels of soluble MUC1 predict a poor clinical response to chemotherapy.
Tumor MUC1 expression analyzed using immunohistochemistry was scored by the percentage of positively stained cells. The data of the present study demonstrated that serum KL-6 correlated with tumor MUC1 expression. The results indicate a potential for serum KL-6 measurements in assessing the local tumor MUC1 status non-invasively. Currently, $>30$ trials for MUC1-targeted therapeutics are being investigated in either early or late phase clinical trials, including the following: ImMucin, which is a $21 \mathrm{mer}$ synthetic vaccine composed of the entire signal peptide domain of the MUC1; tecemotide, which contains 25 amino acids from the variable number tandem repeat region of MUC1; ONT-10, a liposomal vaccine, which contains a unique tumor-specific antigen designed to mimic portions of MUC1; and GO-203, a cell-penetrating peptide-based inhibitor of MUC1 (15,47-49). Tumor heterogeneity is a pertinent obstacle in developing cancer therapeutics. Given that tumor characteristics are likely to differ in primary and relapsed tumors, serum KL-6 based assessments could aid in appropriate patient selection, thereby enabling progress in these MUC1-targeted therapies. Additionally, serum KL-6 levels may allow for real-time monitoring of tumor MUC1 expression during MUC1-targeted treatment. Our group is currently assessing the value of serum KL-6 in monitoring disease progression through a longitudinal study in cancer patients with recurrent ovarian cancer during standard chemotherapy.

One of the limitations for circulating KL-6 as a biomarker for EOC is its specificity for detecting ILDs. In the present study, 2 patients with benign ovarian tumors exhibited relatively high serum KL-6 levels. One patient was previously diagnosed with interstitial pneumonia and had serum KL-6 levels of $1,297 \mathrm{U} / \mathrm{ml}$. This patient was the only one with ILD in the present study. The other patient, who had KL-6 levels of $1,091 \mathrm{U} / \mathrm{ml}$, was a habitual heavy smoker, although it is unclear if smoking contributed to high serum KL-6 levels. Evidently, it may be difficult to interpret serum KL-6 levels in the diagnosis of EOC if the patients had ILDs as a comorbidity. Alternatively, patients with EOC may develop ILDs during the course of chemotherapy or targeted-therapy treatments, making it more complicated when judging the levels of serum KL-6. However, additional measurements of biomarkers for ILDs, such as surfactant proteins A and D (29) may aid in improving the understanding of more complicated cases.

In conclusion, serum KL-6 levels and tumor MUC1 expression were evaluated in patients with $\mathrm{EOC}$ in the present study. It was demonstrated that circulating KL-6 has a better diagnostic value in detecting EOC compared with CA125 in patients suspected of having malignant ovarian tumors. Furthermore, it was revealed that serum KL-6 may be complementary to CA125 at early stages of EOC detection. High levels of circulating KL-6 are associated with a shorter PFS than low levels of circulating KL-6 in patients with EOC. In addition, it was demonstrated that serum KL-6 correlates with tumor MUC1 expression, thus suggesting a potential application for MUC1-targeted therapy in patient selection and treatment monitoring.

\section{Acknowledgements}

The authors would like to thank Dr S. Nagao, Dr N. Iwasa and $\operatorname{Dr}$ T. Nishikawa for their support in sample collection 
during the present study, and Ms. A. Miyara for her technical assistance (Department of Gynecologic Oncology, Saitama Medical University International Medical Center). The Department of Gynecologic Oncology, Saitama Medical University International Medical Center received support from EIDIA Co., Ltd. (grant no. 1705) to conduct the present study.

\section{References}

1. Ryerson AB, Eheman CR, Altekruse SF, Ward JW, Jemal A, Sherman RL, Henley SJ, Holtzman D, Lake A, Noone AM, et al: Annual report to the nation on the status of cancer, 1975-2012, featuring the increasing incidence of liver cancer. Cancer 122: 1312-1237, 2016

2. American Cancer Society: Cancer Facts \& Figures 2015. In: American Cancer Society 2015.

3. Jemal A, Siegel R, Ward E, Hao Y, Xu J, Murray T and Thun MJ: Cancer statistics, 2008. CA Cancer J Clin 58: 71-96, 2008.

4. Trimbos JB, Parmar M, Vergote I, Guthrie D, Bolis G, Colombo N, Vermorken JB, Torri V, Mangioni C, Pecorelli S, et al: International collaborative ovarian neoplasm trial 1 and adjuvant chemotherapy in ovarian neoplasm trial: Two parallel randomized phase III trials of adjuvant chemotherapy in patients with early-stage ovarian carcinoma. J Natl Cancer Inst 95: 105-112, 2003.

5. Committee on the State of the science in ovarian cancer R, Board on Health Care S, Institute of M, National Academies of Sciences E and Medicine: In: Ovarian Cancers: Evolving Paradigms in Research and Care National Academies Press (US) Copyright 2016 by the National Academy of Sciences. All rights reserved. Washington (DC), 2016.

6. Niloff JM, Knapp RC, Schaetzl E, Reynolds C and Bast RC Jr: CA125 antigen levels in obstetric and gynecologic patients. Obstet Gynecol 64: 703-707, 1984.

7. Mor G, Visintin I, Lai Y, Zhao H, Schwartz P, Rutherford T, Yue L, Bray-Ward P and Ward DC: Serum protein markers for early detection of ovarian cancer. Proc Natl Acad Sci USA 102: 7677-7682,2005.

8. Nossov V, Amneus M, Su F, Lang J, Janco JM, Reddy ST and Farias-Eisner R: The early detection of ovarian cancer: From traditional methods to proteomics. Can we really do better than serum CA-125? Am J Obstet Gynecol 199: 215-223, 2008.

9. Luo G, Xiao Z, Long J, Liu Z, Liu L, Liu C, Xu J, Ni Q and Yu X: CA125 is superior to CA19-9 in predicting the resectability of pancreatic cancer. J Gastrointest Surg 17: 2092-2098, 2013.

10. Yerushalmi R, Tyldesley S, Kennecke H, Speers C, Woods R, Knight B and Gelmon KA: Tumor markers in metastatic breast cancer subtypes: Frequency of elevation and correlation with outcome. Ann Oncol 23: 338-345, 2012.

11. Pollán M, Varela G, Torres A, de la Torre M, Ludeña MD, Ortega MD, Pac J, Freixenet J, Gómez G, Sebastián F, et al: Clinical value of p53, c-erbB-2, CEA and CA125 regarding relapse, metastasis and death in resectable non-small cell lung cancer. Int J Cancer 107: 781-790, 2003.

12. Kim DH, Yun HY, Ryu DH, Han HS, Han JH, Yoon SM and Youn SJ: Preoperative CA 125 is significant indicator of curative resection in gastric cancer patients. World J Gastroenterol 21: 1216-1221, 2015

13. Lewis MR, Euscher ED, Deavers MT, Silva EG and Malpica A: Metastatic colorectal adenocarcinoma involving the ovary with elevated serum CA125: A potential diagnostic pitfall. Gynecol Oncol 105: 395-398, 2007.

14. Basal E, Eghbali-Fatourechi GZ, Kalli KR, Hartmann LC, Goodman KM, Goode EL, Kamen BA, Low PS and Knutson KL: Functional folate receptor alpha is elevated in the blood of ovarian cancer patients. PLoS One 4: e6292, 2009.

15. Nath S and Mukherjee P: MUC1: A multifaceted oncoprotein with a key role in cancer progression. Trends Mol Med 20: 332-342, 2014

16. Lau SK, Weiss LM and Chu PG: Differential expression of MUC1, MUC2, and MUC5AC in carcinomas of various sites: An immunohistochemical study. Am J Clin Pathol 122: 61-69, 2004.

17. Deng J, Wang L, Chen H, Li L, Ma Y, Ni J and Li Y: The role of tumour-associated MUC1 in epithelial ovarian cancer metastasis and progression. Cancer Metastasis Rev 32: 535-551, 2013.
18. Wang L, Ma J, Liu F, Yu Q, Chu G, Perkins AC and Li Y: Expression of MUC1 in primary and metastatic human epithelial ovarian cancer and its therapeutic significance. Gynecol Oncol 105: 695-702, 2007

19. Yolken RH, Peterson JA, Vonderfecht SL, Fouts ET, Midthun K and Newburg DS: Human milk mucin inhibits rotavirus replication and prevents experimental gastroenteritis. J Clin Invest 90: 1984-1991, 1992

20. Schroten H, Hanisch FG, Plogmann R, Hacker J, Uhlenbruck G, Nobis-Bosch R and Wahn V: Inhibition of adhesion of S-fimbriated Escherichia coli to buccal epithelial cells by human milk fat globule membrane components: A novel aspect of the protective function of mucins in the nonimmunoglobulin fraction. Infect Immun 60: 2893-2899, 1992.

21. Nicholson S, Bomphray CC, Thomas H, McIndoe A, Barton D, Gore $\mathrm{M}$ and George AJ: A phase I trial of idiotypic vaccination with HMFG1 in ovarian cancer. Cancer Immunol Immunother 53: 809-816, 2004.

22. Verheijen RH, Massuger LF, Benigno BB, Epenetos AA, Lopes A, Soper JT, Markowska J, Vyzula R, Jobling T, Stamp G, et al: Phase III trial of intraperitoneal therapy with yttrium-90-labeled HMFG1 murine monoclonal antibody in patients with epithelial ovarian cancer after a surgically defined complete remission. J Clin Oncol 24: 571-578, 2006.

23. Thathiah A and Carson DD: MT1-MMP mediates MUC1 shedding independent of TACE/ADAM17. Biochem J 382: 363-373, 2004.

24. Carson DD: The cytoplasmic tail of MUC1: A very busy place. Sci Signal 1: pe35, 2008.

25. Levitin F, Stern O, Weiss M, Gil-Henn C, Ziv R, Prokocimer Z, Smorodinsky NI, Rubinstein DB and Wreschner DH: The MUC1 SEA module is a self-cleaving domain. J Biol Chem 280: 33374-33386, 2005.

26. Stahel RA, Gilks WR, Lehmann HP and Schenker T: Third International Workshop on lung tumor and differentiation antigens: Overview of the results of the central data analysis. Int J Cancer Suppl 8: 6-26, 1994

27. Kohno N, Inoue $Y$, Hamada H, Fujioka S, Fujino S, Yokoyama A, Hiwada K, Ueda N and Akiyama M: Difference in sero-diagnostic values among KL-6-associated mucins classified as cluster 9. Int J Cancer Suppl 8: 81-83, 1994.

28. Seko A, Ohkura T, Ideo H and Yamashita K: Novel O-linked glycans containing 6'-sulfo-Gal/GalNAc of MUC1 secreted from human breast cancer YMB-S cells: Possible carbohydrate epitopes of KL-6(MUC1) monoclonal antibody. Glycobiology 22: 181-195, 2012.

29. Ishikawa N, Hattori N, Yokoyama A and Kohno N: Utility of KL-6/MUC1 in the clinical management of interstitial lung diseases. Respir Investig 50: 3-13, 2012.

30. Kohno N, Kyoizumi S, Awaya Y, Fukuhara H, Yamakido M and Akiyama M: New serum indicator of interstitial pneumonitis activity. Sialylated carbohydrate antigen KL-6. Chest 96: 68-73, 1989.

31. Nakao M, Oguri T, Miyazaki M, Uemura T, Takakuwa O, Kunii E, Ohkubo H, Maeno K and Sato S: Diagnosis of ovarian cancers using thoracoscopy: Three case reports and review of the literature. Exp Ther Med 4: 141-145, 2012.

32. Kurosaki A, Hasegawa K, Kato T, Abe K, Hanaoka T, Miyara A, O'Shannessy DJ, Somers EB, Yasuda M, Sekino T and Fujiwara K: Serum folate receptor alpha as a biomarker for ovarian cancer: Implications for diagnosis, prognosis and predicting its local tumor expression. Int J Cancer 138: 1994-2002, 2016.

33. Heintz AP, Odicino F, Maisonneuve P, Quinn MA, Benedet JL, Creasman WT, Ngan HY, Pecorelli S and Beller U: Carcinoma of the ovary. FIGO 26th Annual report on the results of treatment in gynecological cancer. Int J Gynaecol Obstet 95 (Suppl 1): S161-S192, 2006.

34. Youden WJ: Index for rating diagnostic tests. Cancer 3: 32-35, 1950.

35. Rein BJ, Gupta S, Dada R, Safi J, Michener C and Agarwal A: Potential markers for detection and monitoring of ovarian cancer. J Oncol 2011: 475983, 2011

36. Hanaoka T, Hasegawa K, Kato T, Sato S, Kurosaki A, Miyara A, Nagao S, Seki H, Yasuda M and Fujiwara K: Correlation between tumor mesothelin expression and serum mesothelin in patients with epithelial ovarian carcinoma: A potential noninvasive biomarker for mesothelin-targeted therapy. Mol Diagn Ther Feb 3, 2017 (Epub ahead of print).

37. Havrilesky LJ, Whitehead CM, Rubatt JM, Cheek RL, Groelke J, $\mathrm{He}$ Q, Malinowski DP, Fischer TJ and Berchuck A: Evaluation of biomarker panels for early stage ovarian cancer detection and monitoring for disease recurrence. Gynecol Oncol 110: 374-382, 2008. 
38. Pillai K, Pourgholami MH, Chua TC and Morris DL: MUC1 as a potential target in anticancer therapies. Am J Clin Oncol 38: 108-118, 2015

39. Murray A, Willsher P, Price MR, Dixon AR and Robertson JF Evaluation of the IMMULITE BR-MA and CEA assays and comparison with immunoradiometric assays for CA15-3 and CEA in breast cancer. Anticancer Res 17: 1945-1949, 1997.

40. Gion M, Mione R, Leon AE and Dittadi R: Comparison of the diagnostic accuracy of CA27.29 and CA15.3 in primary breast cancer. Clin Chem 45: 630-637, 1999.

41. Engelstaedter V, Heublein S, Schumacher AL, Lenhard M, Engelstaedter H, Andergassen U, Guenthner-Biller M, Kuhn C, Rack B, Kupka M, et al: Mucin-1 and its relation to grade, stage and survival in ovarian carcinoma patients. BMC Cancer 12 600, 2012.

42. Fisken J, Roulston JE, Sturgeon C, Badley RA, Jönrup I, Aspinall $\mathrm{L}$ and Leonard RC: The value of the human milk fat globule membrane antigen HMFG2 in epithelial ovarian cancer monitoring: Comparison with CA125. Br J Cancer 67: 1065-1070, 1993.

43. Kohno N, Akiyama M, Kyoizumi S, Hakoda M, Kobuke K and Yamakido M: Detection of soluble tumor-associated antigens in sera and effusions using novel monoclonal antibodies, KL-3 and KL-6, against lung adenocarcinoma. Jpn J Clin Oncol 18 : 203-216, 1988
44. Ogawa Y, Ishikawa T, Ikeda K, Nakata B, Sawada T, Ogisawa K, Kato Y and Hirakawa K: Evaluation of serum KL-6, a mucin-like glycoprotein, as a tumor marker for breast cancer. Clin Cancer Res 6: 4069-4072, 2000.

45. Gad A, Tanaka E, Matsumoto A, Wahab MA, Serwah Ael-H, Attia F, Ali K, Hassouba H, el-Deeb Ael-R, Ichijyo T, et al: Assessment of KL-6 as a tumor marker in patients with hepatocellular carcinoma. World J Gastroenterol 11: 6607-6612, 2005.

46. Budiu RA, Mantia-Smaldone G, Elishaev E, Chu T, Thaller J, McCabe K, Lenzner D, Edwards RP and Vlad AM: Soluble MUC1 and serum MUC1-specific antibodies are potential prognostic biomarkers for platinum-resistant ovarian cancer. Cancer Immunol Immunother 60: 975-984, 2011.

47. Hasegawa M, Sinha RK, Kumar M, Alam M, Yin L, Raina D, Kharbanda A, Panchamoorthy G, Gupta D, Singh H, et al: Intracellular targeting of the oncogenic MUC1-C protein with a novel GO-203 nanoparticle formulation. Clin Cancer Res 21: 2338-2347, 2015

48. Kovjazin R, Volovitz I, Kundel Y, Rosenbaum E, Medalia G, Horn G, Smorodinsky NI, Brenner B and Carmon L: ImMucin: A novel therapeutic vaccine with promiscuous MHC binding for the treatment of MUC1-expressing tumors. Vaccine 29: 4676-4686, 2011.

49. Sangha R and Butts C: L-BLP25: A peptide vaccine strategy in non small cell lung cancer. Clin Cancer Res 13: s4652-s4654, 2007. 